\title{
ON THE HOMOTOPY AND COHOMOLOGY OF THE CLASSIFYING SPACE OF RIEMANNIAN FOLIATIONS ${ }^{1}$
}

\author{
STEVEN HURDER
}

\begin{abstract}
Let $G$ be a closed subgroup of the general linear group. Let $B \tilde{\Gamma} q$ be the classifying space for $G$-foliated microbundles of rank $q$. (The $G$-foliation is not assumed to be integrable.) The homotopy fiber $F \tilde{\Gamma} \xi$ of the classifying map $\nu$ : $B \tilde{\Gamma}_{G}^{q} \rightarrow B G$ is shown to be $(q-1)$-connected. For the orthogonal group, this implies $F R \Gamma^{q}$ is $(q-1)$-connected. The indecomposable classes in $H^{*}\left(R W_{q}\right)$ therefore are mapped to linearly independent classes in $H^{*}\left(F R \Gamma^{q}\right)$; the indecomposable variable classes are mapped to independently variable classes. Related results on the homotopy groups $\pi_{*}\left(F R \Gamma^{q}\right)$ also follow.
\end{abstract}

1. The main theorem. Let $B R \Gamma^{q}$ be the Haefliger classifying space for Riemannian foliations, $B O(q)$ the classifying space for $O(q)$-bundles and $\nu: B R \Gamma^{q} \rightarrow$ $B O(q)$ the map classifying the normal bundle of the universal $R \Gamma^{q}$-structure on $B R \Gamma^{q}$ [3]. Let $F R \Gamma^{q}$ be the homotopy theoretic fiber of $\nu . H^{*}()$ will denote singular cohomology with real coefficients. In this note we show

THEOREM 1.1. FR $\Gamma^{q}$ is $(q-1)$-connected.

This implies there is a section of $\nu$ over the $q$-skeleton of $B O(q)$, so $\nu^{*}$ : $H^{q}(B O(q)) \rightarrow H^{q}\left(B R \Gamma^{q}\right)$ is injective. On the other hand, the vanishing Theorem of J. Pasternack [9] implies $\nu^{*}: H^{q+1}(B O(q)) \rightarrow H^{q+1}\left(B R \Gamma^{q}\right)$ is the zero map. Theorem 1.1 is therefore the best result possible for $q=4 k+3$. For other $q$, it would be interesting to know whether $F R \Gamma^{q}$ has higher connectivity.

Theorem 1.1 is a special case of a more general result. Let $G \subseteq G l(q, \mathbf{R})$ be a closed subgroup. A foliation on a manifold $M$ is said to be a $G$-foliation [1], [7] if there is given

(i) a model manifold $B$ of dimension $q$ with a $G$-structure on $T B$,

(ii) an open covering $\left\{U_{\alpha}\right\}$ of $M$ and local submersions $\phi_{\alpha}: U_{\alpha} \rightarrow B$ defining the foliation such that the transition functions $\gamma_{\alpha \beta}$ are local $G$-morphisms of $B$.

A $G$-foliation is integrable if it is modeled on $\mathbf{R}^{q}$ with the flat $G$-structure.

A classifying space for $G$-foliations is constructed as follows: Let $\mathscr{N}\left(G, \mathbf{R}^{q}\right)$ denote the total space of the sheaf of local $C^{\infty}$-sections of the bundle $\mathbf{R}^{q} \times$ $G l(q, \mathbf{R}) / G \rightarrow \mathbf{R}^{q}$. This is a (non-Hausdorff) $C^{\infty}$-manifold, with a canonical $G$ structure. Let $\mathcal{S}_{G}$ be the pseudogroup of all local, $C^{\infty}, G$-diffeomorphisms of

Received by the editors October 18, 1979 and, in revised form, February 22, 1980.

AMS (MOS) subject classifications (1970). Primary 57D30, 57D20; Secondary 55F40, 55E15.

Key words and phrases. Classifying spaces, foliations, characteristic classes, minimal models.

${ }^{1}$ This paper is part of the author's doctoral thesis. The author would like to thank his advisor, Franz Kamber, for his guidance and encouragement. 
$\Re\left(G, \mathbf{R}^{q}\right)$ and let $\tilde{\Gamma}_{G}^{q}$ be its associated topological groupoid [1, §2], [3]. Let $B \tilde{\Gamma}_{G}^{q}$ be the Haefliger classifying space of $\tilde{\Gamma}_{G}^{q}$-structures. For $G=O(q)$, we have $B R \Gamma^{q}=$ $B \tilde{\Gamma}_{O(q)}^{q}$ and, in general, $B \tilde{\Gamma}_{G}^{q}$ is the classifying space of $G$-foliations.

There is a natural map $\nu: B \tilde{\Gamma}_{G}^{q} \rightarrow B G$ classifying the normal bundle of the $\tilde{\Gamma}_{G}^{q}$-structure on $B \tilde{\Gamma}_{G}^{q}$. Let $F \tilde{\Gamma}_{G}^{q}$ be the homotopy theoretic fiber of $\nu$.

THEOREM 1.1'. F $\tilde{\Gamma}_{G}^{q}$ is $(q-1)$-connected.

For $G=O(q)$ we recover Theorem 1.1.

There is also a classifying space for integrable $G$-foliations, denoted by $B \Gamma_{G}^{q}$. We let $F \Gamma_{G}^{q}$ denote the homotopy theoretic fiber of $\nu: B \Gamma_{G}^{q} \rightarrow B G$. When $G=\operatorname{Sl}(q, \mathbf{R})$, one can show $B \tilde{\Gamma}_{S l}^{q} \simeq B \Gamma_{S l}^{q}[1$, Remark 4.2], recovering from Theorem 1.1' Haefliger's result that $F \Gamma_{S l}^{q}$ is $(q-1)$-connected.

2. Applications. In this section, we give some consequences of Theorem 1.1'. The proofs of the propositions stated use Sullivan's theory of minimal models [10], and are given in [5].

Theorem 1.1 implies there are many nontrivial Whitehead products in $\pi_{*}\left(B R \Gamma^{q}\right)$ and that many of the secondary characteristic classes map injectively into $H^{*}\left(F R \Gamma^{q}\right)$. To be precise, let $q^{\prime}=[q / 2]$ and $W(\mathfrak{g o}(q))_{q^{\prime}}$ denote the truncated Weil algebra for the orthogonal Lie algebra [7]. The Chern-Weil construction gives a characteristic map $\Delta_{*}: H^{*}\left(W(\mathfrak{g o}(q))_{q^{\prime}}\right) \rightarrow H^{*}\left(F R \Gamma^{q}\right)$. Let $k=[q / 4]+1$ and $m=$ $[(q-1) / 2]$. The set of invariants factors as

$$
H^{*}\left(W(\mathfrak{B o}(q))_{q^{\prime}}\right) \simeq A \otimes \Lambda\left(y_{k}, \ldots, y_{m}\right),
$$

where $A$ is an algebra with all products zero and the second factor is the exterior algebra on generators $y_{j}$ of degree $4 j-1$. The algebra $A$ has an explicit basis, given by 1 and the cocycles $y_{I} p_{J} \in W(\mathfrak{g o}(q))_{q}$, where

$$
y_{I} p_{J}=y_{i_{1}} \cdots y_{i_{j}} p_{1}^{j_{1}} \cdots p_{k=1}^{j_{k-1}}
$$

$1<i_{1}<\cdots<i_{s}<k$ and $l<i_{1} \Rightarrow j_{l}=0$, and $\operatorname{deg} p_{i_{1}} p_{J}>q, \operatorname{deg} p_{J}<q$.

For $q$ even, additional cocycles involving the Euler class must be added to this list [8]. A basis element $y_{I} p_{J} \in A$ is said to be variable if $\operatorname{deg} y_{i_{1}} p_{J}=2 q^{\prime}+1$.

Let $V \subseteq H^{*}\left(W(\mathfrak{g o}(q))_{q^{\prime}}\right)$ be the subspace given by the direct sum

$$
V=A \otimes 1 \oplus 1 \otimes \Lambda\left(y_{k}, \ldots, y_{m}\right) \text {. }
$$

Proposition 2.1. $\Delta_{*}: V \rightarrow H^{*}\left(F R \Gamma^{q}\right)$ is injective, and the variable basis elements in $V$ are mapped to independently variable classes in $H^{*}\left(F R \Gamma^{q}\right)$.

The first statement follows from Theorem 1.1 and the results of F. Kamber and $\mathrm{Ph}$. Tondeur [6, Theorem 6.52]. The variability follows from the examples of $\mathrm{C}$. Lazarov and J. Pasternack [8, Theorem 3.6] combined with Theorem 1.1. Details can be found in [5].

Similar results concerning the homotopy of $F R \Gamma^{q}$ can be shown. Set $\pi^{*}\left(F R \Gamma^{q}\right)$ $=\operatorname{Hom}\left(\pi_{*}\left(F R \Gamma^{q}\right), \mathbf{R}\right)$. Let $\left\langle y_{k}, \ldots, y_{m}\right\rangle$ denote the real vector space spanned by $\left\{y_{k}, \ldots, y_{m}\right\}$. In [5], a vector-space map $h^{\sharp} \circ \zeta: H^{*}\left(W(\mathfrak{g o}(q))_{q^{\prime}}\right) \rightarrow \pi^{*}\left(F R \Gamma^{q}\right)$ is defined, for which 
Proposition 2.2. $h^{\sharp} \circ \zeta: A \oplus\left\langle y_{k}, \ldots, y_{m}\right\rangle \rightarrow \pi^{*}\left(F R \Gamma^{q}\right)$ is injective and the variable basis elements of $A$ are mapped to independently variable classes.

For any commutative cochain algebra $\mathbb{Q}$ there is a vector space $\pi^{*}(\mathscr{Q})$, the dual homotopy of $\mathcal{Q}$, constructed by choosing a minimal model $\mathfrak{T} \rightarrow \mathbb{Q}$, and setting $\pi^{*}(\mathscr{Q})=\Re^{*} /\left(\mathfrak{R}^{+} \cdot \mathfrak{R}^{+}\right)[10]$. The algebra $A$ has trivial products and differential, so for $q=4,6$ or $\geqslant 8$ the vector space $\pi^{*}(A)$ is of finite type but not finite dimensional. There is induced a map $\Delta^{\sharp}: \pi^{*}(A) \rightarrow \pi^{*}\left(F R \Gamma^{q}\right)$, extending $h^{\sharp} \circ \zeta$, for which we have [5]

Proposition 2.3. $\Delta^{\sharp}: \pi^{*}(A) \oplus\left\langle y_{k}, \ldots, y_{m}\right\rangle \rightarrow \pi^{*}\left(F R \Gamma^{q}\right)$ is injective and the variable classes are mapped to independently variable classes.

The following proposition gives our final remark on the homotopy of $F \tilde{\Gamma}_{G}^{q}$. The proof is obvious, using minimal models.

Proposition 2.4. Let $X$ be an n-connected space, $n \geqslant 1$. Then the rational Hurewicz map $\mathcal{H}: \pi_{m}(X) \otimes \mathbf{Q} \rightarrow H_{m}(X ; \mathbf{Q})$ is an isomorphism for $m<2 n$ and an epimorphism for $m=2 n+1$.

Corollary 2.5. $\mathcal{H}: \pi_{m}\left(F \tilde{\Gamma}_{G}^{q}\right) \otimes \mathbf{Q} \rightarrow H_{m}\left(F \tilde{\Gamma}_{G}^{q} ; \mathbf{Q}\right)$ is an isomorphism for $m<2 q$ -2 and an epimorphism for $m=2 q-1$.

3. Proof of Theorem 1.1'. Let $X \subseteq \mathbf{R}^{q}$ be an open subset homotopic to $S^{n}$. When $n$ is zero, we consider $S^{0}$ to consist of a single point. Then $\pi_{n}\left(F \tilde{\Gamma}_{G}^{q}\right) \simeq\left[X, F \tilde{\Gamma}_{G}^{q}\right]$, the set of homotopy classes of maps $f: X \rightarrow F \tilde{\Gamma}_{G}^{q}$. By the Gromov-PhillipsHaefliger Theorem [2], there is a bijection between $\left[X, F \tilde{\Gamma}_{G}^{q}\right]$ and the set of integrable homotopy classes of $G$-foliations on $X$ with trivial $G$-structure. We will show two such foliations on $X$ are integrably homotopic.

Recall that two codimension $q G$-foliations $\mathscr{F}_{0}, \mathscr{F}_{1}$ on $X$ are integrably homotopic if there is a codimension $q G$-foliation $\mathscr{F}$ on $X \times[0,1]$ such that the slices $i_{t}$ : $X \times\{t\} \rightarrow X \times[0,1]$ are transverse to $\mathscr{F}$ for all $t$, and induce $\mathscr{F}_{t}$ for $t=0,1$.

Fix an integer $n$ with $0 \leqslant n<q$. Let $(\theta, r) \in \mathbf{R}^{n+1}$ be polar coordinates, with $\theta \in S^{n}$ and $r \in \mathbf{R}$. For any $a, b \in \mathbf{R}$ with $0<a<b$, define

$$
B(a, b)=\left\{(\theta, r) \in \mathbf{R}^{n+1} \mid a<r<b\right\} \times \mathbf{R}^{q-n-1} .
$$

Set $X=B(0,1)$; then $X \subseteq \mathbf{R}^{q}$ is open and homotopic to $S^{n}$.

A codimension $q G$-foliation on $X$ must be the point foliation with a $G$-structure on the tangent bundle $T X$. The tangent bundle is trivial, so the $G$-structure is characterized by a smooth map $\alpha: X \rightarrow Y$, where $Y$ is the coset space $G l(q, \mathbf{R}) / G$. We denote by $(X, \alpha)$ the $G$-foliation on $X$ with characteristic map $\alpha$. The $G$-structure on $(X, \alpha)$ is trivial if $\alpha$ is homotopic to the constant map with image the identity coset of $Y$. For two $G$-foliations $\left(X, \alpha_{0}\right)$ and $\left(X, \alpha_{1}\right)$ with trivial $G$-structures, it is apparent that $\alpha_{0}$ and $\alpha_{1}$ are homotopic.

To prove the theorem, it will suffice to show that if $\alpha_{0}$ and $\alpha_{1}$ are homotopic, then there is an integrable homotopy from $\left(X, \alpha_{0}\right)$ to $\left(X, \alpha_{1}\right)$. To do this, we will construct three integrable homotopies, on $X \times[0,1], X \times[1,2]$ and $X \times[2,3]$ which combine to give the desired integrable homotopy. 
Step 1. Choose a monotone, $C^{\infty}$-function

$$
\phi:[0,1] \rightarrow[1 / 2,1] \text { with } \phi(t)= \begin{cases}1 & \text { for } t<1 / 4 \\ 1 / 2 & \text { for } t>3 / 4\end{cases}
$$

Define $H: X \times[0,1] \rightarrow X$ by

$$
H_{t}(\theta, r, v)=(\theta, \phi(t) \cdot(r-1 / 2)+1 / 2, v) .
$$

For each $t, H_{t}$ is a submersion; $H_{0}$ is the identity and $H_{1}$ maps $X$ to a subannulus of $X$. Also, $H_{t}$ is constant with respect to $t$ for $t$ near 0 or 1 .

Define a $G$-structure on $X$ by $\alpha_{0}^{\prime}=\alpha_{0} \circ H_{1}: X \times\{1\} \rightarrow Y$. Then the submersion $H: X \times I \rightarrow\left(X, \alpha_{0}\right)$ defines a $G$-foliation on $X \times[0,1]$ which is an integrable homotopy from $\left(X, \alpha_{0}\right)$ to $\left(X, \alpha_{0}^{\prime}\right)$.

Step 2. Define $H^{\prime \prime}: X \times[2,3] \rightarrow X$ by $H_{t}^{\prime \prime}=H_{3-t}$. Define a $G$-structure on $X$ by setting $\alpha_{1}^{\prime}=\alpha_{1} \circ H_{2}^{\prime \prime}$. Then the submersion $H^{\prime \prime}: X \times[2,3] \rightarrow\left(X, \alpha_{1}\right)$ defines a $G$-foliation which is an integrable homotopy from $\left(X, \alpha_{1}^{\prime}\right)$ to $\left(X, \alpha_{1}\right)$.

Step 3. We next produce an integrable homotopy from $\left(X, \alpha_{0}^{\prime}\right)$ to $\left(X, \alpha_{1}^{\prime}\right)$ by constructing a $G$-foliation $(X, \alpha)$ and a submersion $H^{\prime}: X \times[1,2] \rightarrow(X, \alpha)$ so that $\alpha_{0}^{\prime}=\alpha \circ H_{1}^{\prime}$ and $\alpha_{1}^{\prime}=\alpha \circ H_{2}^{\prime}$.

Define functions $f_{0}$ and $f_{1}$ as follows

$$
\begin{aligned}
& f_{0}: B(5 / 8,1) \rightarrow B(0,3 / 4) \quad \text { by } f_{0}(\theta, r, v)=(\theta, 2 r-5 / 4, v), \\
& f_{1}: B(0,3 / 8) \rightarrow B(1 / 4,1) \text { by } f_{1}(\theta, r, v)=(\theta, 2 r+1 / 4, v) .
\end{aligned}
$$

Note that $f_{0}$ maps $B(3 / 4,1)$ to the image of $H_{1}$ and $f_{1}$ maps $B(0,1 / 4)$ to the image of $\mathrm{H}_{2}^{\prime \prime}$.

There are inclusions

$$
\begin{aligned}
& i_{0}: S^{n} \times\{3 / 4\} \times \mathbf{R}^{n-q-1} \subseteq B(5 / 8,1), \\
& i_{1}: S^{n} \times\{1 / 4\} \times \mathbf{R}^{n-q-1} \subseteq B(0,3 / 8)
\end{aligned}
$$

and the composites $\alpha_{0} \circ f_{0} \circ i_{0}$ and $\alpha_{1} \circ f_{1} \circ i_{1}$ are homotopic by assumption. Therefore, there exists a smooth extension

$$
\tilde{\alpha}: S^{n} \times[1 / 4,3 / 4] \times \mathbf{R}^{n-q-1}=\overline{B(1 / 4,3 / 4)} \rightarrow Y
$$

of $\alpha_{0} \circ f_{0} \circ i_{0} \cup \alpha_{1} \circ f_{1} \circ i_{1}$. We define a smooth map $\alpha: X \rightarrow Y$ by

$$
\alpha= \begin{cases}\alpha_{0} \circ f_{0} & \text { on } B(3 / 4,1), \\ \tilde{\alpha} & \text { on } \overline{B(1 / 4,3 / 4)} \\ \alpha_{1} \circ f_{1} & \text { on } B(0,1 / 4)\end{cases}
$$

Finally, we construct the submersion $H^{\prime}: X \times[1,2] \rightarrow X$. Choose a monotone, $C^{\infty}$-function $\varphi:[1,2] \rightarrow[0,3]$ with

$$
\varphi(t)= \begin{cases}3 & \text { for } t<5 / 4 \\ 0 & \text { for } t>7 / 4\end{cases}
$$

Then $H^{\prime}$ at time $t$ is given by

$$
H_{t}^{\prime}(\theta, r, v)=(\theta, 1 / 4(r+\varphi(t)), v) .
$$

The map $H^{\prime}$ has the effect of sliding the image of $X$ from image $H_{1}$ to image $H_{2}^{\prime \prime}$ as $t$ varies from 1 to 2 . 
Let $X \times[1,2]$ have the $G$-structure defined by the submersion $H^{\prime}: X \times[1,2] \rightarrow$ $(X, \alpha)$. This gives an integrable homotopy from $\left(X, \alpha \circ H_{1}^{\prime}\right)$ to $\left(X, \alpha \circ H_{2}^{\prime}\right)$. A straightforward check shows that $f_{0} \circ H_{1}^{\prime}=H_{1}$ and $f_{1} \circ H_{2}^{\prime}=H_{2}^{\prime \prime}$. This implies $\alpha_{0}^{\prime}=\alpha \circ H_{1}^{\prime}$ and $\alpha_{1}^{\prime}=\alpha \circ H_{2}^{\prime}$, which finishes Step 3 and the proof of Theorem 1.1'.

\section{REFERENCES}

1. T. Duchamp, Characteristic invariants of $G$-foliations, $\mathrm{Ph}$. D. thesis, University of Illinois, Urbana, III., 1976.

2. A. Haefliger, Feuilletages sur les variétés ourvertes, Topology 9 (1970), 183-194.

3. __ Homotopy and integrability, Lecture Notes in Math., vol. 197, Springer-Verlag, Berlin and New York, 1971, pp. 133-163.

4. __ Whitehead products and differential forms, Lecture Notes in Math., vol. 652, SpringerVerlag, Berlin and New York, pp. 13-24.

5. S. Hurder, Dual homotopy invariants of $\mathrm{G}$-foliations, $\mathrm{Ph}$. D. Thesis, University of Illinois, Urbana, Ill., 1980.

6. F. Kamber and $\mathrm{Ph}$. Tondeur, Non-trivial invariants of homogeneous foliated bundles, Ann. Sci. École Norm. Sup. 8 (1975), 433-486.

7. , G-foliations and their characteristic classes, Bull. Amer. Math. Soc. 84 (1978), 1086-1124.

8. C. Lazarov and J. Pasternack, Secondary characteristic classes for Riemannian foliations, J. Differential Geometry 11 (1976), 365-385; Residues and characteristic classes for Riemannian foliations, J. Differential Geometry 11 (1976), 599-612.

9. J. Pasternack, Foliations and compact Lie groups actions, Comment. Math. Helv. 46 (1971), 467-477.

10. D. Sullivan, Infinitesimal computations in topology, Inst. Hautes Études Sci. Publ. Math. 47 (1977), 269-331.

Department of Mathematics, University of Illinois at Urbana-Champaign, Urbana, Illinois 61801

Current address: School of Mathematics, Institute for Advanced Study, Princeton, New Jersey 08540 